\title{
Análise da contaminação fúngica em amostras de Cassia acutifolia Delile (sene) e Peumus boldus (Molina) Lyons (boldo-do-Chile) comercializadas na cidade de Campinas, Brasil
}

\author{
Liliana de O. Rocha ${ }^{1}$, Maria Magali S. R. Soares ${ }^{1 *}$, Cristiana Leslie Corrêa ${ }^{1}$ \\ ${ }^{1}$ Faculdade de Ciências Farmacêuticas, Pontifícia Universidade Católica de Campinas
}

- Correspondência:

M.M.S.R. Soares.

Laboratório de Microbiologia e

Imunologia.

Faculdade de Ciências Farmacêuticas.

Pontifícia Universidade Católica de

Campinas.

Centro de Ciências da Vida.

Av. John Boyd Dunlop, Jardim

Ipaussurama, sn.

13059-740, Campinas, SP, Brasil.

E-mail:magali_soares@uol.com.br.
A sociedade atual tem buscado a fitoterapia como um importante recurso terapêutico, sendo a avaliação da qualidade microbiológica destes produtos um requisito essencial, considerando a sua origem. Deste modo, o objetivo da pesquisa foi realizar a contagem e a identificação de fungos filamentosos em 20 amostras de folhas de Cassia acutifolia Delile (sene) e de Peumus boldus (Molina) Lyons (boldo-do-Chile) comercializadas em farmácias de manipulação e mercados da cidade de Campinas, Brasil, usando as técnicas microbiológicas clássicas. Os resultados obtidos evidenciaram que 45\% das amostras analisadas se situavam fora dos padrões estabelecidos pela Organização Mundial da Saúde (OMS). Não foram observadas diferenças significativas na análise quantitativa da contaminação fúngica entre amostras comercializadas em farmácias de manipulação e mercados, tanto para o boldo-doChile como para a sene. Os fungos identificados nestas amostras foram: Aspergillus, Penicillium, Phoma, Cladosporium, Trichoderma, Rhizopus, Mucor, Aureobasidium pullulans, Mycelia sterilia, Acremonium e Monilia sitophila. Estes resultados demonstraram o baixo nivel de qualidade desses fitoterápicos, pois, além do elevado número de amostras contaminadas, foram identificados fungos de gêneros produtores de micotoxinas, como o Aspergillus e o Penicillium. Verifica-se a urgência na realização de adequado controle de qualidade microbiológico dos fitoterápicos e de implantação de fiscalização efetiva, para garantir a segurança e eficácia destes produtos.
Unitermos

- Produtos fitoterápicos

- Controle de qualidade microbiológico

- Fungos

- Cassia acutifolia

- Peumus boldus

\section{INTRODUÇÃO}

A sociedade atual tem buscado na natureza hábitos mais saudáveis de vida. Essa tendência, associada ao alto custo dos medicamentos, tem levado ao aumento progressivo da produção e utilização de medicamentos fitoterápicos e, conseqüentemente, à preocupação em relação a qualidade destes produtos, já que as contaminações, as 
falsificações e as adulterações são freqüentes (Araújo, Ohara, 2000; Brandão et al., 2001).

A prática da fitoterapia segura encontra uma série de dificuldades, que vão, desde a identificação correta do material botânico utilizado, à inexistência de estudos de segurança, eficácia e qualidade de grande parte das plantas. A qualidade implica controle e nele estão envolvidos experimentos nos quais se insere o controle microbiológico, cujo principal objetivo é analisar a contaminação por microrganismos, entre os quais se encontram os fungos filamentosos (Brandão et al., 1998; Bugno et al., 2002; Santos et al., 1995).

Como os fungos podem ser dispersos pelo ar atmosférico, pode ocorrer contaminação das plantas, antes e após sua colheita, como também durante o processamento. Entre os principais gêneros detectados no Brasil, destacam-se: Cladosporium, Fusarium, Aspergillus, Penicillium e Rhizopus. A presença destes fungos em fitoterápicos pode ser prejudicial à saúde humana, uma vez que estes podem causar micotoxicoses, quando introduzidos por via oral, ou outras doenças, quando inalados (Corrêa, 1998; Kneifel et al., 2001; Lacaz et al., 2002).

De acordo com a RDC n ${ }^{\circ} 48$ de 16 de março de 2004, os medicamentos fitoterápicos devem ser registrados conforme as normas da Agência Nacional de Vigilância Sanitária (ANVISA), sendo a pesquisa de contaminantes microbiológicos também contemplada nas recomendações da Organização Mundial da Saúde (OMS) (ANVISA, 2004; Garcia et al., [entre 1993 e 2003]; WHO, 1992).

Os objetivos deste trabalho foram: avaliar a presença, quantificar, isolar e identificar fungos filamentosos em amostras de folhas de sene e boldo-do-Chile obtidos de mercados e farmácias de manipulação na cidade de Campinas, visando contribuir para a melhoria do perfil de qualidade destes fitoterápicos.

\section{MATERIAL E MÉTODOS}

\section{Amostragem}

Foram adquiridas 20 amostras de folhas inteiras de Cassia acutifolia Delile (sene) e 20 de Peumus boldus (Molina) Lyons (boldo-do-Chile) nas suas embalagens originais, em farmácias de manipulação ( $n=10$ /espécie) e em mercados ( $\mathrm{n}=10$ /espécie) da cidade de Campinas, São Paulo, Brasil.

\section{Determinação quantitativa e identificação dos contaminantes fúngicos}

Pesaram-se $10 \mathrm{~g}$ de cada amostra, que foi macerada em gral, previamente esterilizado. Posteriormente, esta foi diluída em $90 \mathrm{~mL}$ de tampão fosfato $\mathrm{pH}$ 7,0, e submetida aos processos de agitação e filtração em gaze. Em segui$\mathrm{da}$, foram pipetados $10 \mathrm{~mL}$ do filtrado para erlenmeyer contendo $90 \mathrm{~mL}$ de tampão fosfato $\mathrm{pH} 7,0$, realizando 3 diluições decimais sucessivas (Pinto et al., 2000; Santos et al.,1995).

Foram transferidos $0,1 \mathrm{~mL}$ de cada diluição para placas de Petri contendo meio de cultura Ágar Sabouraud Dextrose (ASD) (Merck KgaA, Alemanha). Incubaramse as placas à temperatura de cerca de $25^{\circ} \mathrm{C}$, por 5 dias (Pinto et al.,2000; Santos et al.,1995). Após este período, realizou-se a contagem de colônias (UFC/g). Posteriormente, estas foram repicadas em tubos de ensaio contendo Ágar Sabouraud Dextrose(ASD) e incubadas a $25^{\circ} \mathrm{C}$, por 5 dias. Após o crescimento, os fungos foram identificados macro e microscopicamente, de acordo com técnicas clássicas de Microbiologia (Lacaz et al., 2002; Larone, 2002).

\section{Análise estatística}

Foi utilizado o teste t pareado para comparar a quantidade de amostras contaminadas nos mercados e nas farmácias de manipulação e a contaminação (quantitativa) encontrada nos fitoterápicos boldo-do-Chile e sene. O nível de significância adotado foi de $10 \%(\mathrm{p}<0,10)$. Utilizou-se o programa Instat ${ }^{\circledR}$ (GraphPad Software), versão 3.05.

\section{RESULTADOS E DISCUSSÃO}

Em fitoterápicos, o limite máximo permitido, pela Organização Mundial da Saúde, para a carga de fungos filamentosos é de $5 \times 10^{2} \mathrm{UFC} / \mathrm{g}$ (Santos et al.,1995).

De acordo com os resultados encontrados, 92,5\% das amostras apresentaram contaminação fúngica, sendo que, $45 \%$ (18 amostras) apresentaram níveis acima do limite preconizado e $47,5 \%$ (19 amostras) mostraram-se dentro do limite (Tabela I).

Observou-se elevado número de amostras de fitoterápicos contaminadas tanto em farmácias de manipulação como nos mercados municipais. Em relação às folhas de sene, a contaminação foi maior nas amostras provenientes de farmácias de manipulação $(50 \%)$ e, para o boldodo-Chile, o crescimento fúngico foi maior nas amostras comercializadas em mercados (60\%) (Tabela I). Em adição, nas amostras de sene e boldo-do-Chile provenientes de mercados, os níveis de contaminação variaram de $1,0 \times 10^{1} \mathrm{UFC} / \mathrm{g}$ a $7,37 \times 10^{3} \mathrm{UFC} / \mathrm{g}$ (Tabela II) e de $5,0 \times 10^{1} \mathrm{UFC} / \mathrm{g}$ a $1,9 \times 10^{3} \mathrm{UFC} / \mathrm{g}$ (Tabela III), respectivamente. A carga fúngica para amostras de sene obtidas de 
farmácias de manipulação variaram de $1,1 \times 10^{2} \mathrm{UFC} / \mathrm{g}$ a $6,2 \times 10^{4} \mathrm{UFC} / \mathrm{g}$ e para boldo-do-Chile variaram de $1,0 \times 10^{1} \mathrm{UFC} / \mathrm{g}$ a $5,0 \times 10^{3} \mathrm{UFC} / \mathrm{g}$ (Tabelas II e III). Alguns bolores encontrados nestas amostras apresentam-se, comumente, dispersos no ar (Kneifel et al., 2001), desempenhando papel importante na etiologia das alergias respiratórias e intoxicações por micotoxinas, como a aflatoxina produzida pelo gênero Aspergillus (Corrêa, 1998; Lacaz et al., 2002).

Os resultados referentes às 10 amostras de folhas de sene provenientes de mercados, demonstraram que $30 \%$ estavam acima do limite máximo (Santos et al., 1995). Na análise realizada, foram encontrados fungos dos gêneros: Rhizopus, Aureobasidium, Mucor e Mycelia

TABELA I - Confronto da contaminação fúngica das amostras de sene e boldo-do-Chile adquiridas na cidade de Campinas, São Paulo, com os limites de tolerância preconizados pela OMS

\begin{tabular}{|c|c|c|c|c|c|}
\hline \multirow{3}{*}{$\begin{array}{l}\text { Nível de } \\
\text { contaminação } \\
\text { das amostras }\end{array}$} & \multicolumn{2}{|c|}{$\operatorname{sene}^{4}$ n $(\%)$} & \multicolumn{2}{|c|}{ boldo-do-Chile ${ }^{4}$ (\%) } & \multirow[t]{3}{*}{ TOTALn (\%) } \\
\hline & \multicolumn{4}{|c|}{ Locais de aquisição } & \\
\hline & Mercado $^{2}$ & $\begin{array}{l}\text { Farmácias de } \\
\text { Manipulação }^{2}\end{array}$ & Mercado $^{3}$ & $\begin{array}{l}\text { Farmácias de } \\
\text { Manipulação }^{3}\end{array}$ & \\
\hline Superior ao LMP ${ }^{1}$ & $3(30)$ & $5(50)$ & $6(60)$ & $4(40)$ & $18(45)$ \\
\hline Em acordo com LMP ${ }^{1}$ & $7(70)$ & $4(40)$ & $3(30)$ & $5(50)$ & $19(47,5)$ \\
\hline Ausente & $0(0)$ & $1(10)$ & $1(10)$ & $1(10)$ & $3(7,5)$ \\
\hline
\end{tabular}

${ }^{1}$ LMP- Limite Máximo Permitido pela OMS (5x102 UFC/g) (Santos et al., 1995); ${ }^{2}$ Comparação dos resultados de contaminação fúngica entre as amostras de sene coletadas em mercado e em farmácias de manipulação: $\mathrm{p}>0,10(\mathrm{t}=0,000$; $2 \mathrm{gl}) ;{ }^{3}$ Comparação dos resultados de contaminação fúngica entre as amostras de boldo-do-Chile coletadas em mercado e em farmácias de manipulação: $\mathrm{p}>0,10(\mathrm{t}=0,000 ; 2 \mathrm{gl}) ;{ }^{4}$ Comparação dos resultados de contaminação fúngica entre as amostras de sene e boldo-do-Chile: $\mathrm{p}>0,10(\mathrm{t}=0,000 ; 5 \mathrm{gl})$.

TABELA II - Confronto da contagem fúngica total (UFC/g) e identificação das espécies presentes em amostras de sene comercializadas em mercados e farmácias de manipulação de Campinas, São Paulo

\begin{tabular}{|c|c|c|c|c|}
\hline \multirow[b]{2}{*}{ Amostras } & \multicolumn{2}{|c|}{ Mercado } & \multicolumn{2}{|c|}{ Farmácias de manipulação } \\
\hline & $\begin{array}{l}\text { Contagem } \\
\text { total }(\text { UFC/g) }\end{array}$ & Fungos & $\begin{array}{l}\text { Contagem } \\
\text { total }(\text { UFC/g) }\end{array}$ & Fungos \\
\hline 1 & $1,0 \times 10^{1}$ & Rhizopus & 0 & - \\
\hline 2 & $1,0 \times 10^{1}$ & Mycelia sterilia & $1,1 \times 10^{2}$ & Rhizopus \\
\hline 3 & $1,0 \times 10^{1}$ & Rhizopus & $1,2 \times 10^{2}$ & $\begin{array}{l}\text { Rhizopus } \\
\text { Aspergillus }\end{array}$ \\
\hline 4 & $1,0 \times 10^{1}$ & Rhizopus & $3,6 \times 10^{2}$ & $\begin{array}{c}\text { Rhizopus } \\
\text { Aureobasidium pullulans }\end{array}$ \\
\hline 5 & $5,5 \times 10^{1}$ & Rhizopus & $3,7 \times 10^{2}$ & $\begin{array}{c}\text { Penicillium } \\
\text { Mycelia sterilia }\end{array}$ \\
\hline 6 & $5,5 \times 10^{1}$ & Rhizopus & $7,5 \times 10^{2}$ & $\begin{array}{c}\text { Rhizopus } \\
\text { Aureobasidium pullulans }\end{array}$ \\
\hline 7 & $1,7 \times 10^{2}$ & $\begin{array}{c}\text { Rhizopus } \\
\text { Aureobasidium pullulans }\end{array}$ & $8,3 \times 10^{2}$ & Aureobasidium pullulans \\
\hline 8 & $9,6 \times 10^{2}$ & $\begin{array}{c}\text { Mucor } \\
\text { Aureobasidium pullulans }\end{array}$ & $1,2 \times 10^{3}$ & $\begin{array}{c}\text { Rhizopus } \\
\text { Aureobasidium pullulans }\end{array}$ \\
\hline 9 & $1,6 \times 10^{3}$ & $\begin{array}{c}\text { Mucor } \\
\text { Aureobasidium pullulans }\end{array}$ & $2,4 \times 10^{3}$ & $\begin{array}{c}\text { Rhizopus } \\
\text { Aureobasidium pullulans }\end{array}$ \\
\hline 10 & $7,37 \times 10^{3}$ & $\begin{array}{c}\text { Rhizopus } \\
\text { Aureobasidium pullulans }\end{array}$ & $6,2 \times 10^{4}$ & $\begin{array}{c}\text { Monilia sitophila } \\
\text { Rhizopus } \\
\text { Aureobasidium pullulans }\end{array}$ \\
\hline
\end{tabular}




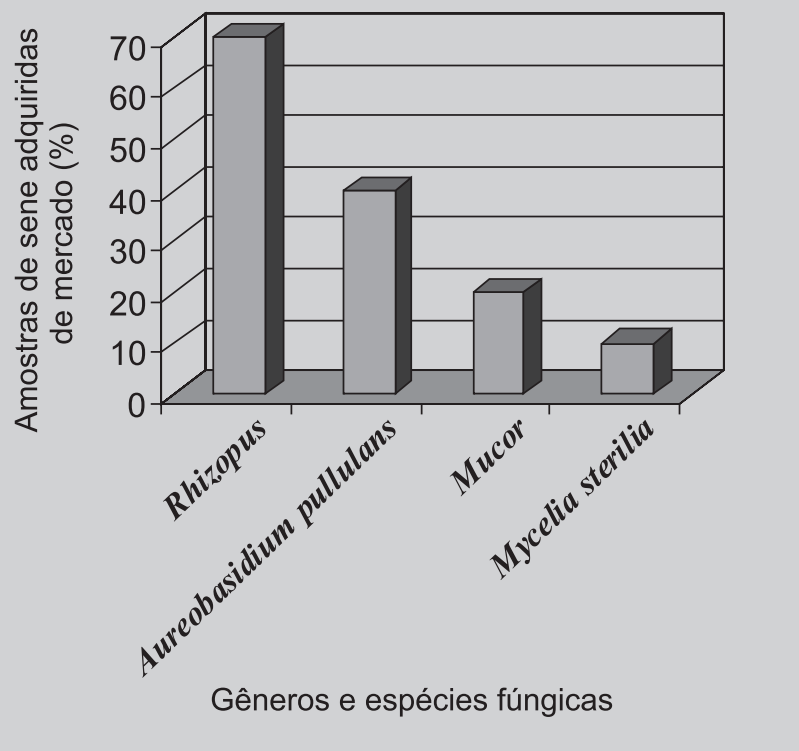

FIGURA 1 - Freqüência relativa de gêneros e espécies fúngicas em amostras de sene comercializadas em mercados de Campinas.

(Tabelas I e II, Figura 1). Nas amostras de sene obtidas de farmácias de manipulação, foram detectados fungos dos gêneros Rhizopus, Aspergillus e Penicillium e as espécies Aureobasidium pullulans, Mycelia sterilia e Monilia sitophila (Tabelas I e II, Figura 2).

Dentre as amostras de boldo-do-Chile, provenientes de mercados, $60 \%$ apresentaram contaminação acima do limite permitido pela OMS (WHO, 1992), sendo identificados os contaminantes dos gêneros: Mucor, Penicillium, Cladosporium, Phoma, Acremonium e Aspergillus, e as espécies: Aureobasidium pullulans e Mycelia sterilia (Tabelas I e III, Figura 3). No entanto, a partir das amostras obtidas de farmácias de manipulação, verificou-se que $40 \%$ apresentaram contaminação acima do preconizado, tendo-se isolado: Rhizopus, Aspergillus, Mycelia sterilia, Aureobasidium pullulans, Penicillium, e Trichoderma (Tabelas I e III, Figura 4).

Para as duas espécies vegetais, verificou-se que 12 $(60 \%)$ amostras de sene e 10 (50\%) de boldo-do-Chile apresentaram carga fúngica inferior a $5 \times 10^{2} \mathrm{UFC} / \mathrm{g}$ (Tabelas II e III).

Considerando o parâmetro da OMS $\left(5,0 \times 10^{2} \mathrm{UFC} /\right.$ g), pode-se dizer que 8 amostras de sene (40\%) estão acima do mesmo, sendo que $5(25 \%)$ adquiridas em farmácias de manipulação e 10 (50\%) amostras de boldo-doChile se encontram na mesma situação, sendo 6 (30\%) provenientes de mercados (Tabelas II e III).

Em apenas uma amostra de sene o nível de contaminação foi superior a $10^{4} \mathrm{UFC} / \mathrm{g}$, sendo que os gêneros e as

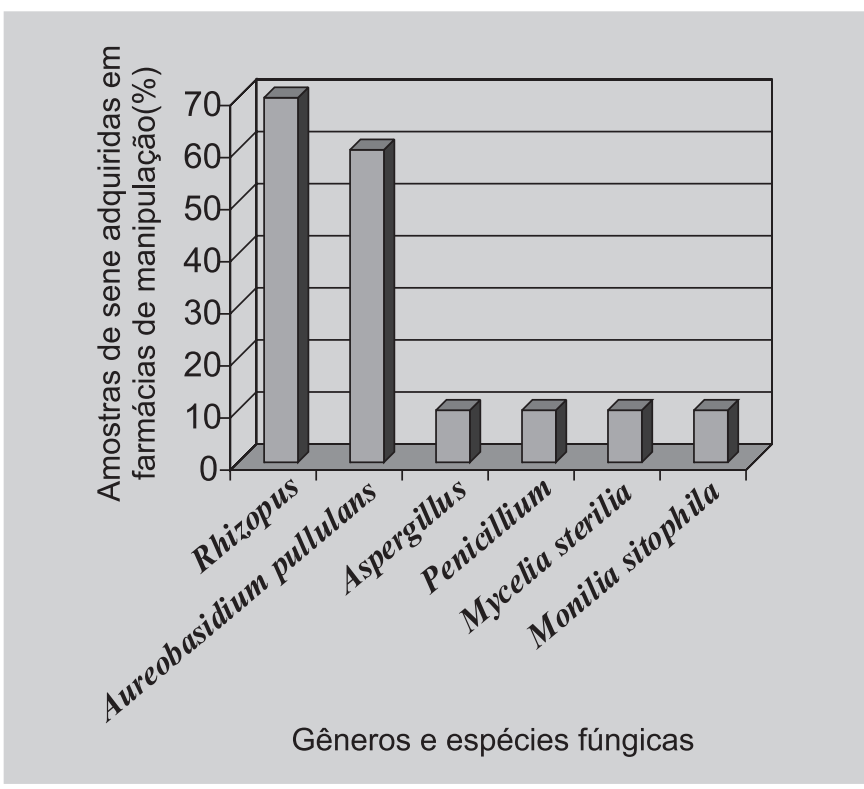

FIGURA 2 - Freqüência relativa de gêneros e espécies fúngicas em amostras de sene comercializadas em farmácias de manipulação de Campinas.

espécies fúngicas encontradas nesta amostra foram: Monilia sitophila; Rhizopus e Aureobasidium pullulans.

Entretanto, de acordo com a análise estatística (Tabela I) verificou-se que não houve diferença significativa nos níveis de contaminação fúngica (análise quantitativa) entre os locais de aquisição de amostras ( $p>0,10)$, assim como entre as duas espécies vegetais estudadas $(p>0,10)$.

Os fitoterápicos, de maneira geral, são suscetíveis à contaminação fúngica durante o processo de plantio e colheita. Além disso, a manipulação e o armazenamento inadequado destes produtos, que podem ocorrer tanto nas farmácias de manipulação como nas indústrias que produzem os fitoterápicos, podem ser fonte de contaminação secundária.

Os resultados demonstram o baixo nível de qualidade destes fitoterápicos, pois além do número considerável de amostras contaminadas $(92,5 \%)$, foram identificados bolores, que podem produzir micotoxinas, destacando-se os gêneros Aspergillus (10\% das amostras) e Penicillium (7,5\% das amostras). Estes foram encontrados em folhas de sene provenientes de farmácias de manipulação e em folhas de boldo-do-Chile obtidas tanto de farmácias de manipulação como de mercados. É importante ressaltar que o boldo-do-Chile é um fitoterápico amplamente utilizado pela população e neste houve maior contaminação por fungos toxigênicos. Foram identificados os gêneros: Phoma, Cladosporium e Trichoderma, que também são capazes de produzir micotoxinas. Deste modo, a utilização desses fitoterápicos apresenta risco adicional ao consumidor, que pode estar ingerindo micotoxinas (Corrêa, 1998). 
TABELA III - Confronto da contagem total (UFC/g) e identificação de fungos presentes em amostras de boldo-do-Chile comercializadas em mercados e farmácias de manipulação de Campinas

\begin{tabular}{|c|c|c|c|c|}
\hline \multirow[b]{2}{*}{ Amostras } & \multicolumn{2}{|c|}{ Mercado } & \multicolumn{2}{|c|}{ Farmácias de manipulação } \\
\hline & $\begin{array}{l}\text { Contagem } \\
\text { total (UFC/g) }\end{array}$ & Fungos & $\begin{array}{l}\text { Contagem } \\
\text { total (UFC/g) }\end{array}$ & Fungos \\
\hline 1 & 0 & - & 0 & - \\
\hline 2 & $5,0 \times 10^{1}$ & Aspergillus & $1,0 \times 10^{1}$ & Trichoderma \\
\hline 3 & $1,4 \times 10^{2}$ & $\begin{array}{c}\text { Mycelia sterilia } \\
\text { Mucor }\end{array}$ & $2,0 \times 10^{1}$ & Rhizopus \\
\hline 4 & $1,7 \times 10^{2}$ & $\begin{array}{c}\text { Aureobasidium pullulans } \\
\text { Mycelia sterilia } \\
\text { Phoma } \\
\text { Cladosporium }\end{array}$ & $1,3 \times 10^{2}$ & Aureobasidium pullulans \\
\hline 5 & $7,4 \times 10^{2}$ & $\begin{array}{c}\text { Penicillium } \\
\text { Aureobasidium pullulans }\end{array}$ & $4,0 \times 10^{2}$ & $\begin{array}{l}\text { Mycelia sterilia } \\
\text { Penicillium }\end{array}$ \\
\hline 6 & $7,8 \times 10^{2}$ & $\begin{array}{l}\text { Mycelia sterilia } \\
\text { Aureobasidium pullulans }\end{array}$ & $4,1 \times 10^{2}$ & Mycelia sterilia \\
\hline 7 & $1,0 \times 10^{3}$ & $\begin{array}{c}\text { Mycelia sterilia } \\
\text { Aureobasidium pullulans }\end{array}$ & $7,9 \times 10^{2}$ & $\begin{array}{l}\text { Mycelia sterilia } \\
\text { Aureobasidium pullulans }\end{array}$ \\
\hline 8 & $1,0 \times 10^{3}$ & Aureobasidium pullulans & $1,0 \times 10^{3}$ & $\begin{array}{c}\text { Rhizopus } \\
\text { Aspergillus }\end{array}$ \\
\hline 9 & $1,1 \times 10^{3}$ & $\begin{array}{c}\text { Mycelia sterilia } \\
\text { Aspergillus }\end{array}$ & $3,0 \times 10^{3}$ & Mycelia sterilia \\
\hline 10 & $1,9 \times 10^{3}$ & $\begin{array}{l}\text { Phoma } \\
\text { Acremonium }\end{array}$ & $5,0 \times 10^{3}$ & Aureobasidium pullulans \\
\hline
\end{tabular}

As micotoxinas podem ocasionar intoxicações agudas ou crônicas quando da ingestão de produtos contaminados por bolores, mesmo na forma de chá, pois são termoestáveis. É importante ressaltar que as aflatoxinas produzidas pelo gênero Aspergillus possuem elevado ponto de fusão (ao redor de $269^{\circ} \mathrm{C}$ ) e apresentam potencial carcinogênico (WHO, 1979). São classificadas, segundo o IARC (International Agency for Research on Cancer), no grupo 1, definido como carcinogênico para o homem (Abou-Arab et al.,1999; Araújo, Ohara, 2000; Corrêa, 1998; IARC, 1993).

Alguns bolores encontrados, neste estudo (espécies de Aspergillus e Penicillium), podem causar doenças, como alergias, além de problemas graves em imunossuprimidos. Também, representam risco para quem manipula tais fitoterápicos, tendo em vista a possível inalação durante o processo produtivo (Lacaz et al., 2002; Marques, 1998).

Os demais contaminantes fúngicos encontrados nas amostras são considerados, principalmente, contaminantes saprófitas; no entanto, também podem causar doenças, como o Rhizopus e o Mucor, mais preocupantes em indivíduos imunossuprimidos, e o Acremonium, que está associado a doenças como micetomas (Lacaz et al., 2002;

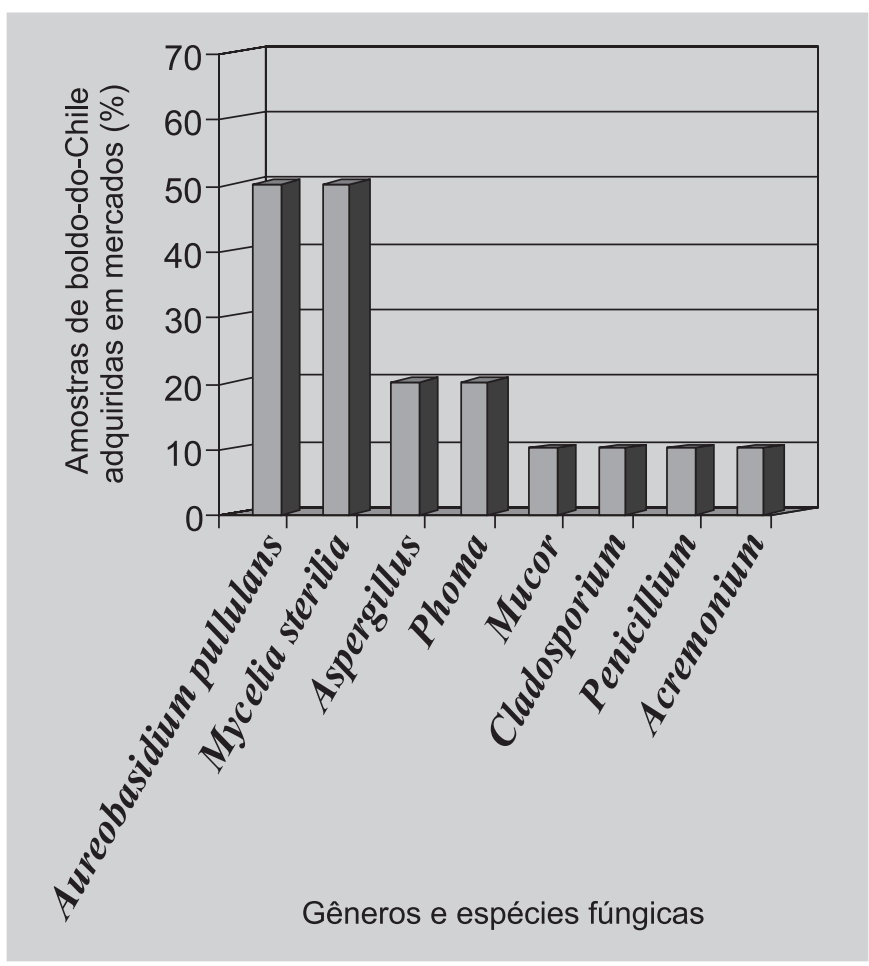

FIGURA 3 - Freqüência relativa de gêneros e espécies fúngicas em amostras de boldo-do-Chile comercializadas em mercados de Campinas. 


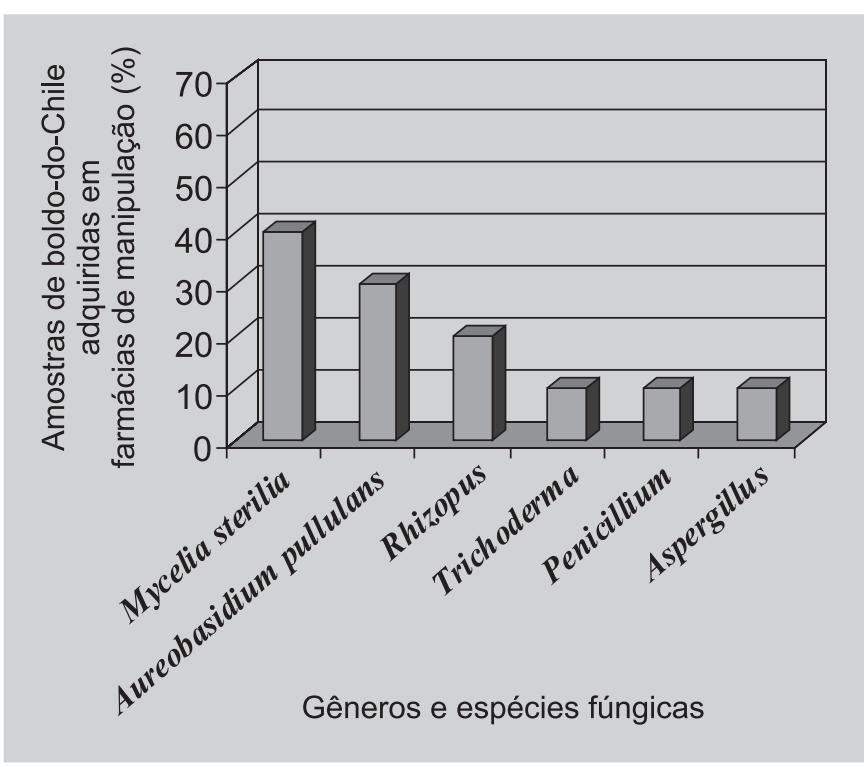

FIGURA 4 - Freqüência relativa de gêneros e espécies fúngicas em amostras de boldo-do- Chile comercializadas em farmácias de manipulação de Campinas.

Larone, 2002; Marques, 1998).

As publicações referentes à identificação e quantificação de fungos em fitoterápicos, no Brasil, são escassas. Santos et al. (1995) e Bugno et al. (2002), igualmente constataram contaminação por Aspergillus e Penicillium, entre outros, em chás para infusão, pós (guaraná), extrato fluido, comprimidos e cápsulas (Bugno et al., 2002; Santos et al., 1995). No entanto, mostram perfis semelhantes àqueles de outros países.

Em Portugal, Martins et al. (2001) verificaram a presença de fungos filamentosos, como: Aspergillus, Penicillium, Absidia, Mucor, Cladosporium e Paecilomyces, em 62 amostras de 7 diferentes fitoterápicos. Resultados semelhantes foram vistos por Abou-Arab et al. (1999), no Egito, e por Efuntoye (1996), na Nigéria.

Os objetivos e resultados deste estudo reforçam a necessidade do controle microbiológico de qualidade em fitoterápicos, em concordância com a legislação atual (ANVISA, 2004), que visa garantir a segurança, eficácia e qualidade destes medicamentos.

\section{ABSTRACT}

Analysis of the fungal contamination in Cassia acutifolia Delile (sene) and Peumus boldus (Molina) Lyons (boldo-do-Chile) sold in Campinas, Brazil

The consumption of medicinal plants has increased during the last years. The purpose of this study was to evaluate and identify fungi specimens present in samples of Cassia acutifolia Delile (20) (sene) and Peumus boldus (Molina) Lyons (Boldo-do-Chile) (20), that were purchased in drugstores and markets of Campinas, Brazil, by usual methods. The results showed that $45 \%$ of samples did not fit the minimum quality standards recommended by the World Health Organization (WHO). No differences were observed in the quantitative analysis between the samples sold at the drugstores and at the markets. The identified genera and species of fungi were: Aspergillus, Penicillium, Phoma, Cladosporium, Trichoderma, Rhizopus, Mucor, Aureobasidium pullulans, Mycelia sterilia, Acremonium and Monilia sitophila. Considering the level of contamination and the presence of Aspergillus and Penicillium, which are able to produce mycotoxins, there is an urgent need for quality control of the phytotherapic products in order to assure their efficacy and safety.

UNITERMS: Medicinal plants. Quality control. Moulds. Cassia acutifolia Delile. Peumus boldus(Molina) Lyons.

\section{REFERÊNCIAS BIBLIOGRÁFICAS}

ABOU-ARAB, A.A.K.; KAWTHER, M.S.; TANTAWY, M.E.E.; BADEAA, R.J.; KHAYRIA, N. Quality estimation of some contaminants in commonly used medicinal plants in the Egyptian market. Food Chem., v.67, p.357-363, 1999.

ARAÚJO, A.L.A.; OHARA, M.T. Qualidade microbiológica de drogas vegetais comercializadas em feira de São Paulo e de infusos derivados.Rev. Bras. Cienc. Farm., v.36, p.129-137, 2000.

BRANDÃO, M.G.L.; FREIRE, N.; SOARES, C.D.V. Vigilância de fitoterápicos de Minas Gerais. Verificação da qualidade de diferentes amostras comerciais de camomila. Cad. Saúde Pública, v.14, p.613-616, 1998.

BRANDÃO, M.G.L.; OLIVEIRA, P.; MOREIRA, R.A.; ALVES, R.M.S.; VIEIRA, M.T.; CAMPOS, L.M.M. Qualidade de amostras comerciais e produtos fitoterápicos: Drogas inscritas na farmacopéia brasileira. Infarma, v.13, p.60-61, 2001.

BRASIL.Agência Nacional de Vigilância Sanitária. Resolução $\mathrm{RDC}^{\circ}$ 48, de 16 de março de 2004. Dispõe sobre o registro de medicamentos fitoterápicos. Diário Oficial da União, Poder Executivo, Brasília, DF, 18 mar. 2004. 
BUGNO, A.; MATOS, D.; PINTO, T.J.A. Contaminação fúngica em plabntas medicinais. Rev. Bras. Cienc. Farm., v.38, supl.1, p.87, 2002.

CORRÊA, B. Micotoxinas humanas e micetismos. In: ZAITZ, C.; CAMPBELL, I.; MARQUES, S.A.; RUIZ, L.R.B.; SOUZA, V.M., eds. Compêndio de micologia médica. Rio de Janeiro: Medsi, 1998. cap. 27, p.339-346.

EFUNTOYE, M.O. Fungi associated with herbal drug plants during storage. Mycophatologia, v.136, p.115-118, 1996.

GARCIA, E. S.; SILVA, A. C. P.; GILBERT, B.; CORRÊA, C. B.; CAVALHEIRO, M. V. S.; SANTOS, R. R.; TOMASSINI, T. Biodiversidade: perspectivas e oportunidades tecnológicas. Base de Dados Tropical, [entre 1993 e 2003]. Disponível em: < http:// www.bdt.fat.org.br/publicacoes/padct/bio/cap10>. Acesso em: 30 jul. 2002.

\section{INTERNATIONAL AGENCY FOR RESEARCH ON} CANCER. Aflatoxins, v. 56, p. 245, 1993. Disponível em: $<$ http://monographs.iarc.fr/htdocs/monographs/vol156/09afl.htm>. Acesso em: 11 maio 2004.

KNEIFEL, W.; CZECH, E.; KOPP, B. Microbial contamination of medicinal plants - a review. Planta Méd., v.68, p. 5-15, 2001.

LACAZ, C.S.; PORTO, E.; MARTINS, J.E.C.; VACCARI, E.M.H.; MELO, N.T. Tratado de micologia médica. 9.ed. São Paulo: Sarvier, 2002. p. 15-829.
LARONE, D.H. Medically important fungi. A guide to identification. 3.ed. Washington: American Society for Microbiology, 1995. p.107-206.

MARQUES, S.A. Micoses em imunossuprimidos. In: ZAITZ, C.; CAMPBELL, I.; MARQUES, S.A.; RUIZ, L.R.B.; SOUZA, V.M. , eds. Compêndio de micologia médica. Rio de Janeiro: Medsi, 1998. cap.26, p.325-338.

MARTINS, H.M.; MARTINS, M.L.; DIAS, M.I.; BERNARDO,F. Evaluation of microbiological quality of medicinal plants used in natural infusions. Int. J. Food Microbiol., v. 68, p.149-153, 2001.

PINTO, T.J.A.; KANEKO, T.M.; OHARA, M.T. Controle biológico de qualidade de produtos farmacêuticos, correlatos e cosméticos. São Paulo: Atheneu, 2000. p.75-95.

SANTOS, P.R.V.; OLIVEIRA, A.C.X.; TOMASSINI, T.C.B. Controle microbiológico de produtos fitoterápicos. Rev. Farm. Bioquim.Univ.São Paulo, v.31, p.35-38, 1995.

WORLD HEALTH ORGANIZATION. Mycotoxins. Geneva, WHO, 1979. (Environmental Crtiteria, 11). Disponível em: $<\mathrm{http}$ ://www.inchem.org/documents/ehc/ ehc/ehc011.htm>. Acesso em: 15 set. 2004.

WORLD HEALTH ORGANIZATION. Quality control methods for medicinal plants materials. Geneva: WHO, 1992. $122 \mathrm{p}$.

Recebido para publicação em 24 de agosto de 2004. Aceito para publicação em 24 de dezembro de 2004. 